\title{
Medulloblastoma, WNT-Activated
}

National Cancer Institute

\section{Source}

National Cancer Institute. Medulloblastoma, WNT-Activated. NCI Thesaurus. Code C129440.

A molecular subtype of medulloblastoma associated with activation of the WNT pathway. TP53 mutations may be present or absent. WNT pathway activation in medulloblastomas is associated with good outcome. 